\title{
Management of Hypercholesterolemia, Appropriateness of Therapeutic Approaches and New Drugs in Patients with High Cardiovascular Risk
}

\author{
Enrico Agabiti Rosei $^{1} \cdot$ Massimo Salvetti $^{1}$ (D)
}

Received: 5 April 2016/Accepted: 25 April 2016/Published online: 27 August 2016

(c) The Author(s) 2016. This article is published with open access at Springerlink.com

\begin{abstract}
Control of lipid levels is one of the most effective strategies for cardiovascular (CV) event prevention. In fact, many clinical trials have clearly demonstrated that lowdensity lipoprotein cholesterol (LDL-C) lowering, primarily with statins, reduces major CV events and mortality. The evidence from these trials has been useful in designing the cholesterol treatment guidelines, which are mainly aimed at preventing and managing cardiovascular disease (CVD). However, available data indicate that a large proportion of patients fail to achieve lipid goals, and this is particularly frequent in patients at high or very high $\mathrm{CV}$ risk. Furthermore, owing to side effects, a significant percentage of patients cannot tolerate statin treatment. Hence, researchers have focused their attention on novel LDL-C-lowering agents that act via mechanisms distinct from that of statins. Among the new compounds under investigation, the monoclonal antibodies to proprotein convertase subtilisin/kexin type 9 (PCSK9) seem particularly promising, having recently been shown to be well tolerated and highly effective at lowering LDL-C, with a possible effect on the occurrence of CV events. Currently, alirocumab is approved by the US Food and Drug Administration (FDA) as an adjunct to diet and maximally tolerated statin therapy for use in adults with heterozygous familial hypercholesterolemia $(\mathrm{FH})$ or those with atherosclerotic $\mathrm{CV}$ disease who require additional LDL-C lowering; it has also been
\end{abstract}

Enrico Agabiti Rosei

enrico.agabitirosei@unibs.it

Massimo Salvetti

massimo.salvetti@unibs.it

1 Clinica Medica, Department of Clinical and Experimental Sciences, University of Brescia, c/o 2a Medicina Spedali Civili di Brescia, Piazzale Spedali Civili 1, 25123 Brescia, Italy recently approved by the European Medicines Agency (EMA) for use in patients with heterozygous $\mathrm{FH}$, non-familial hypercholesterolemia or mixed dyslipidemia in whom statins are ineffective or not tolerated. Evolocumab is approved by the FDA as an adjunct to diet and maximally tolerated statins for adults with hetero- and homozygous FH and those with atherosclerotic $\mathrm{CV}$ disease who require additional lowering of LDL-C, and by the EMA in adults with primary hypercholesterolemia or mixed dyslipidemia, as an adjunct to diet, in combination with a statin or a statin with other lipid lowering therapies in patients unable to reach LDL-C goals with the maximum tolerated dose of a statin; alone or in combination with other lipid lowering therapies in patients who are statin-intolerant, or those for whom a statin is contraindicated. Evolocumab is also indicated in adults and adolescents aged 12 years and over with homozygous familial hypercholesterolemia in combination with other lipid-lowering therapies.

Keywords PCSK9 antibodies - Evolocumab .

Alirocumab - Bococizumab - Dyslipidemia .

Cardiovascular risk

\section{Introduction}

Cardiovascular disease (CVD) remains the leading cause of death and a major cause of disability in western countries, and it is also becoming increasingly common in developing countries $[1,2]$. The causes of CVD are multifactorial; cardiovascular $(\mathrm{CV})$ risk is strongly influenced by lifestyle, especially an unhealthy diet, tobacco use and sedentary habits, which are modifiable $[1,2]$. A relevant role is also played by other modifiable risk factors, such as high blood pressure, diabetes and dyslipidemias, and by non- 
modifiable factors like age and male gender $[1,2]$. In the last few decades preventive strategies based on lifestyle changes and control of $\mathrm{CV}$ risk factors provided significant benefits; in the MONICA project, which took place during the 1980s and 1990s [3], the relationship between changes in risk factor scores and CV event rates was substantial, with the changes in risk factors explaining almost half the variation in event rates.

Control of lipid levels is one of the most effective strategies for CVD prevention. Epidemiologic data have demonstrated the crucial role of dyslipidemia, especially hypercholesterolemia, in the development of CVD [4]. It is well understood that accumulation of cholesterol-rich low density lipoproteins over time leads to formation of lipid-laden foam cells and proliferation of atherosclerotic lesions, increasing the risk of CVD [5]. Reduction of cholesterol levels may be achieved by implementing lifestyle changes, and use of pharmacological interventions such as statins, bile acid sequestrants, nicotinic acid and cholesterol absorption inhibitors. Available evidence confirms that reducing plasma low-density lipoprotein cholesterol (LDL-C) reduces CVD risk, and it is generally accepted that the reduction of LDL-C levels is of prime importance in the prevention of CVD; most current guidelines on the management of dyslipidemia or the prevention of CVD identify LDL-C as a primary treatment target [1, 6-9]. Many clinical trials have clearly demonstrated that cholesterol lowering, primarily with statins, reduces major vascular events and mortality [10].

The data obtained in more than 25 years of clinical trials have established statins as the preferred therapy for the prevention and treatment of atherosclerotic CVD. Due to their demonstrated efficacy and safety, they have become first-line therapy when lipids are elevated despite appropriate lifestyle changes. After the demonstration of the favourable effect of statins on $\mathrm{CV}$ outcomes when used in both primary and secondary prevention strategies [11], subsequent findings suggested the possibility that intensive treatment with statins and attainment of lower LDL-C targets might lead to greater benefits [10]; more recently, the results of the double-blind, randomized IMPROVE-IT study indicated that simvastatin and ezetimibe combination therapy improved overall cardiovascular outcomes by significantly lowering median timeweighted average LDL-C level $(53.7 \mathrm{mg} / \mathrm{dL})$ when compared to simvastatin monotherapy $(69.5 \mathrm{mg} / \mathrm{dL}$; $\mathrm{p}<0.001$ ), thus adding further weight to the idea that more intensive LDL-C-lowering results in better CV outcomes [12]. Our aim is to present an overview of the management of hypercholesterolemia, with a focus on current unmet needs and the novel therapies currently being investigated that may fill those gaps.

\section{Achievement of Treatment Targets}

The current European Society of Cardiology and European Atherosclerosis Society (ESC/EAS) guidelines [9] recommend treatment targets for dyslipidemia that are primarily based on results from clinical trials; since in nearly all lipid-lowering trials the LDL-C level has been used as an indicator of response to therapy, the recommendations centre on LDL-C. The ESC/EAS guidelines recommend modulating the intensity of the preventive intervention according to the total level of $\mathrm{CV}$ risk, where moderate risk is defined as a calculated SCORE (Systematic Coronary Risk Estimation) of $\geq 1$ and $<5 \%$ at 10 years, high risk is defined as a calculated 10-year SCORE of $\geq 5$ and $<10 \%$ and/or a markedly elevated single risk factor (e.g., familial dyslipidemia, severe hypertension), and very high risk is defined as documented CVD, previous myocardial infarction, acute coronary syndrome, coronary revascularisation, ischaemic stroke, or peripheral arterial disease; and/or type 2 diabetes or patients with type 1 diabetes with target organ damage; and/or a calculated 10-year SCORE of $\geq 10 \%$. For LDL-C, these guidelines recommend a target of $<100 \mathrm{mg} / \mathrm{dL}$ in high risk patients (level of evidence: class IIa, level A) and a target of $<70 \mathrm{mg} / \mathrm{dL}$ for very high risk patients (class I, level A); a level of less than $115 \mathrm{mg} / \mathrm{dL}$ is suggested for patients at moderate risk (class IIa, level C) [9]. Non-high-density lipoprotein cholesterol (non-HDL-C) (which includes very low density lipoproteins, intermediate-density lipoproteins, lipoprotein (a) and other remnants) and apolipoprotein B (apoB) have also been used as secondary targets for the treatment of CVD [9].

Recent data further reinforce the concept that achieving lower LDL-C targets may confer even greater benefits. A recent meta-analysis on individual patient data from eight randomized statin trials found that patients who achieve very low LDL-C levels $(<50 \mathrm{mg} / \mathrm{dL})$ have a lower risk of CV events than do those achieving moderately low levels (Fig. 1) [13]. Of note, patients achieving an LDL-C level of $<50 \mathrm{mg} / \mathrm{dL}$ had a statistically significant decrease in the risk of CV events even versus patients with an LDL-C level between 75 and $<100 \mathrm{mg} / \mathrm{dL}$ [adjusted hazard ratio (HR):

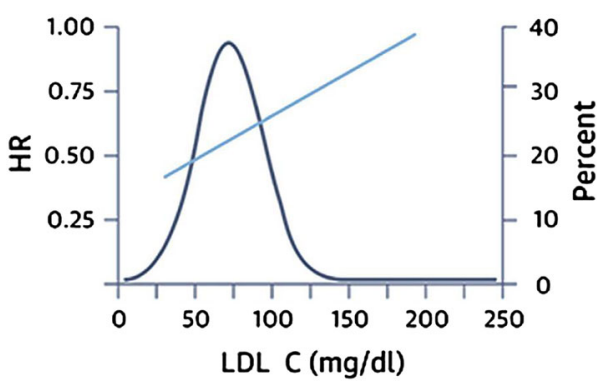

Fig. 1 Risk of major cardiovascular events versus LDL-C levels Reproduced with permission from Boekholdt et al. [13] 
$0.81 ; 95 \%$ confidence interval (CI): 0.70-0.95]. However, the study also showed large inter-individual variability in the reduction in levels of LDL-C, non-HDL-C, and apoB achieved with fixed-dose statins; more than $40 \%$ of patients receiving high-dose statin therapy (defined as atorvastatin $80 \mathrm{mg}$ or rosuvastatin $20 \mathrm{mg}$ ) did not achieve the LDL-C target of $<70 \mathrm{mg} / \mathrm{dL}$, while 11.7 and $33.7 \%$ did not achieve non-HDL-C targets of $<130$ and $<100 \mathrm{mg} / \mathrm{dL}$, respectively, and 14.7 and $35.7 \%$ did not achieve apoB targets of $<100$ and $<80 \mathrm{mg} / \mathrm{dL}$, respectively [13]. The study underlines the fact that a large proportion of patients fail to achieve the lipid targets suggested by current guidelines and, at the same time, seems to indicate that insufficient control of LDL-C levels in these patients might expose them to an increased risk of CV events. Inadequate control of lipid levels seems to be even more common in everyday practice: the results of EUROASPIRE IV [14], a cross-sectional study reporting data from 24 European countries, have shown that less than $20 \%$ of patients with known coronary artery disease attain the LDL$\mathrm{C}$ levels indicated by European guidelines. These results are in accordance with earlier multinational surveys of secondary prevention practice conducted in Europe and in other parts of the world [15-17].

It is therefore evident that attainment of lipid targets is infrequent, both in everyday practice and in clinical trials, even in very high risk patients in whom LDL-C should be more aggressively reduced, such as those with severe primary hypercholesterolemia, including familial hypercholesterolemia $(\mathrm{FH}$; an autosomal dominant disorder involving mutations primarily in the LDL receptor gene, characterised by defective plasma LDL-C catabolism and LDL-C levels of at least two times normal $[18,19])$. As recognized by current European guidelines, even with maximum doses of lipid-lowering therapies, one cannot expect to achieve optimal lipid levels $(<70 \mathrm{mg} / \mathrm{dL})$ in patients with very high baseline LDL-C levels; the maximum reduction in LDL-C that can be achieved without inducing side effects is therefore considered an acceptable option [9]. In these patients, generally maximum doses of atorvastatin (80 mg [20]) or rosuvastatin (40 mg [21]) are required, and combination therapy is often considered [9]. Despite this, only a minority of these patients achieve the suggested targets; in a large, cross-sectional study of 1249 patients with heterozygous $\mathrm{FH}$ (heFH) in the Netherlands, only $21 \%$ of patients achieved an LDL-C target of $<2.5 \mathrm{mmol} / \mathrm{L}(<100 \mathrm{mg} / \mathrm{dL})$ [22].

\section{Reasons for Unmet Needs}

In addition to the aforementioned reasons, lack of attainment of lipid targets may be explained by several other factors. For example, non-adherence to cholesterol- lowering medication is a significant clinical problem [23]. A study performed in Italy showed that the initial statin prescription was not followed by another prescription in $47.5 \%$ of newly-treated patients [24], confirming previous results from a meta-analysis indicating that as many as $60 \%$ of patients may stop their statin therapy within 6 months of treatment initiation [25].

The reasons for non-adherence are incompletely understood. Among the possible explanations, the asymptomatic nature of the disease is likely to play a role. In fact, it has been demonstrated that patient perception of the disease may influence adherence, and patients with a personal history of heart disease are more adherent to statin therapy than patients with no personal history [23].

Side effects of treatment will certainly affect adherence. A recognized side effect of statins is statin-myositis, defined as muscle symptoms in association with a substantially elevated serum creatine kinase (CK) concentration; albeit serious, statin-associated myopathy is a rare side effect, affecting 1 in 1000 to 1 in 10,000 people on statin treatment [26]. In contrast, statin-associated muscle symptoms (SAMS) in which CK concentrations are normal or only slightly elevated, are much more common: data from patient registries indicate a prevalence of 7-29\% [27-31]. SAMS are likely to be one of the principal reasons for the $75 \%$ statin discontinuation rates seen within 2 years of treatment initiation, as well as a significant contributor to statin non-adherence rates, therefore contributing to the lack of control of cholesterol levels [26]. EAS recently published a consensus document which presents an overview of current understanding of the pathophysiology of SAMS, and provides guidance for diagnosis and management of this condition [26]. The panel proposed to identify the occurrence of SAMS via the presence of typical statin-related myalgia (muscle pain/ aching), and the temporal relationship between the presence of SAMS and statin initiation, discontinuation and statin re-exposure following discontinuation [26]. The use of the maximum tolerated statin dose combined with nonstatin lipid-lowering therapies is recommended in patients with SAMS and uncontrolled hypercholesterolemia [26]. Among these additional non-statin lipid-lowering therapies, ezetimibe is proposed as a first-line drug, in view of its ability to reduce LDL-C by $15-20 \%$ with few side effects $[26,32]$, and of the demonstration of a significant effectiveness in reducing $\mathrm{CV}$ outcomes in the IMPROVEIT study, in which the addition of ezetimibe $10 \mathrm{mg}$ to simvastatin $40 \mathrm{mg}$ significantly reduced the primary composite $\mathrm{CV}$ end point versus simvastatin monotherapy (Kaplan-Meier event rate at 7 years: 32.7 vs. $34.7 \%$; HR: 0.936 ; $95 \%$ CI $0.89-0.99 ; \mathrm{p}=0.016$ ) [12]. The EAS panel concluded that with proper recognition of SAMS and adherence to a structured work-up, patients with clinically 
relevant SAMS can be offered treatment regimens that will address their CVD risk in a satisfactory manner.

\section{Novel Therapies}

Given current unmet needs, there is a major requirement for additional therapeutic options for lipid lowering. Therefore, researchers have focused their attention on the development of novel therapies that address the limitations of currently available treatments for hypercholesterolemia and that might have good tolerability as well as the potential to lower serum LDL-C levels below those achievable with statin therapy alone.

\subsection{Proprotein Convertase Subtilisin/Kexin Type 9 (PCSK9)}

In 2003, Seidah et al. identified proprotein convertase subtilisin/kexin type 9 (PCSK9), a secreted 692-amino acid protein belonging to the proprotein convertase family that post-transcriptionally promotes a reduction in LDL receptor levels [33]. Secreted from the liver, circulating PCSK9 binds to the LDL receptor and is subsequently internalized with the receptor, promoting its cellular degradation (2). In the same year, Abifadel and coworkers [34] identified gain of function (GOF) mutations of PCSK9 as a third cause of $\mathrm{FH}$, after exclusion of mutations in the LDL receptor apoB. Subsequently, in 2004 and 2005, other PCSK9 GOF mutations were described in $\mathrm{FH}$ patients from the UK, USA and Norway [18, 19, 35]. GOF mutations are rare, accounting for a minority of cases of $\mathrm{FH}$; available data indicate that these mutations are not only associated with high cholesterol levels, but also with a greater atherosclerotic burden [36], similar to what is observed with the more traditional mutations in the LDL receptor and apoB that lead to FH. Interestingly, several loss-offunction (LOF) mutations in PCSK9 have been found associated with lower plasma LDL-C and lower prevalence of coronary artery disease. Furthermore, the longitudinal data of the atherosclerosis risk in communities (ARIC) study [37] have shown that compared with non-carriers, the presence of the LOF mutations Y142X and C679X in African Americans was associated with a 28 and $88 \%$ reduction in LDL-C and risk of CHD, respectively. In the same study, the R46L mutation in Caucasians was associated with a reduction in LDL-C of $15 \%$, and a reduction in the risk of $\mathrm{CHD}$ of $47 \%$, versus non-carriers [37]. In a meta-analysis published in 2010 [38], 46L allele carriers had a $12 \%$ reduction in LDL-C and a $28 \%$ reduction in risk of ischemic heart disease. Taken together, these results suggest that inhibition of PCSK9 may be a possible treatment option for patients with uncontrolled LDL-C and high $\mathrm{CV}$ risk.

\subsection{Alirocumab}

Alirocumab (formerly REGN727/SAR236553) is a PCSK9 inhibitor which has been approved by the US Food and Drug Administration (FDA) for use as an adjunct to diet and maximally tolerated statin therapy in adult patients with heterozygous FH or those with atherosclerotic CVD who require additional lowering of LDL-C. It is a fully human monoclonal antibody against PCSK9 administered by subcutaneous injection, and it blocks the ability of PCSK9 to bind surface LDL receptor [39]. The efficacy of alirocumab in reducing LDL-C has been investigated in several studies. Stein and co-workers published the first clear demonstration of the LDL-C-lowering effect of antibodies to PCSK9 in humans in a phase I, multilevel, single ascending dose study in which $0.3,1,3,6$ and $12 \mathrm{mg} / \mathrm{kg}$ doses of alirocumab were administered intravenously in healthy subjects [39]. A significant, dose dependent reduction in LDL-C was observed (reduction ranging from -28 to $-65 \%$ vs placebo), with a plateau effect at 3,6 and $12 \mathrm{mg} / \mathrm{kg}$, suggesting system saturation (i.e., complete binding of circulating PCSK9). The duration of LDL-C lowering was dose-dependent, with prolonged reductions (up to 64 days) seen with the higher doses, and a relationship between the reduction in free PCSK9 levels and the decrease in LDL-C levels following alirocumab administration was seen [39]. The results of a phase I study in which alirocumab was administered by subcutaneous injection (at doses of 50, 100, 150 or $250 \mathrm{mg}$ ) in healthy volunteers was also described [39]; the study demonstrated the efficacy of alirocumab in reducing LDL-C (reduction ranging from -32.5 to $-45.7 \%$ vs placebo). Both studies indicated that no additional lowering of LDL-C occurs once all of the available plasma PCSK9 is bound to alirocumab. However, higher doses of alirocumab prolong the duration of the LDL-C-lowering effect, as newly released PCSK9 binds to the excess drug. Stein et al. also described the results of treatment with multiple subcutaneous doses of alirocumab in adults with heFH $(\mathrm{n}=21)$, and in patients with non-familial hypercholesterolemia $(n=30)$, all of whom were receiving treatment with atorvastatin, and in patients with non-familial hypercholesterolemia treated with modified diet alone $(n=10)$ [39]. No difference in the percent reduction of LDL-C or in the duration of the reduction was observed between patients with or without $\mathrm{FH}$, suggesting that PCSK9 inhibition was able to overcome the adverse functional effects of the genetic mutation in the LDL receptor present in FH. Similarly, the percent reduction in LDL-C was not different whether or not patients were taking atorvastatin [39]. In a pooled analysis 


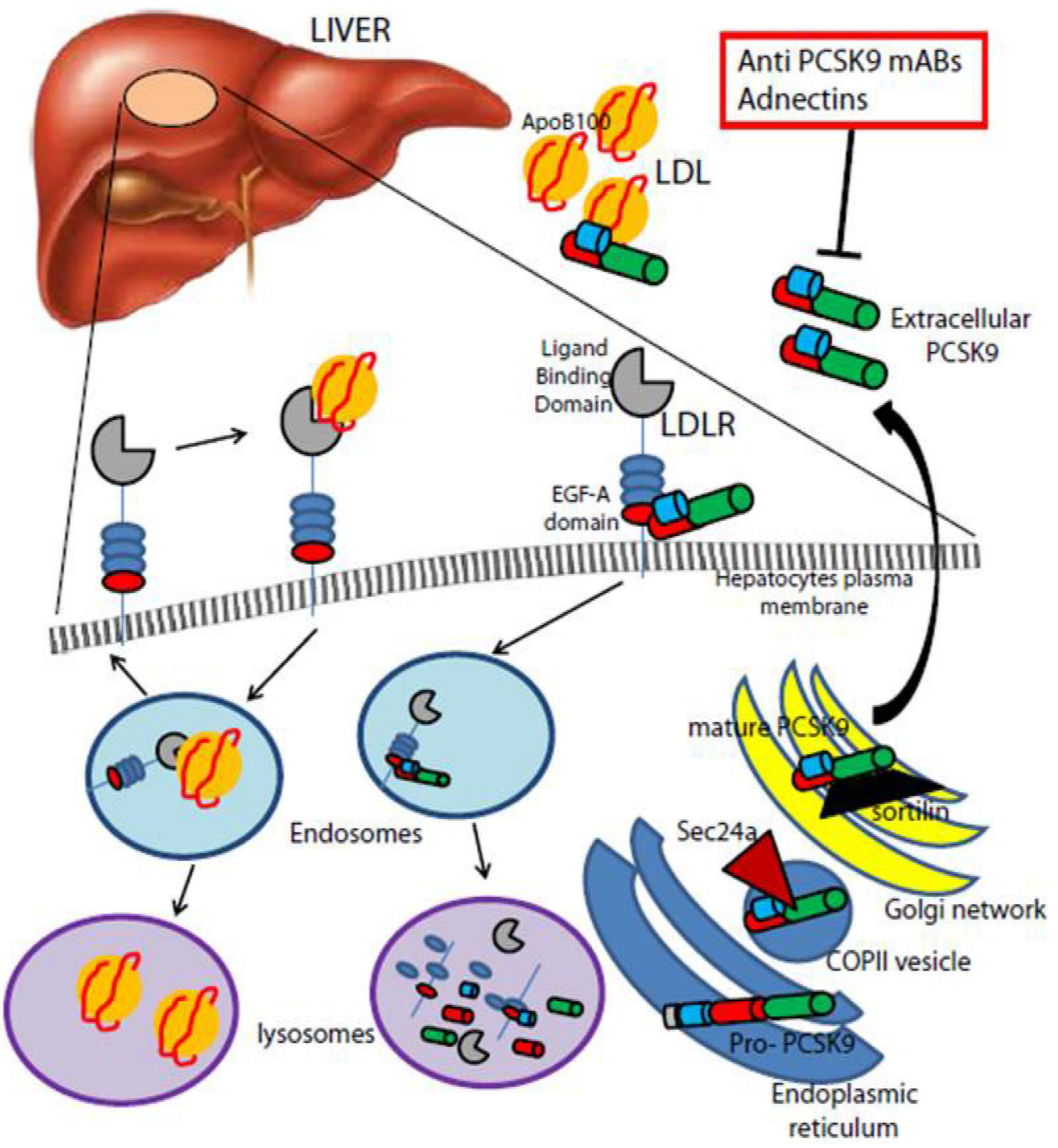

2 PCSK9-mediated degradation of the LDL receptor. The secreted PCSK9 binds the epidermal growth factor-like repeat A domain of the LDL receptor present on the plasma membrane of hepatocytes. The complex undergoes clathrin-mediated endocytosis and is then escorted to lysosomes for degradation, in contrast to the physiological

(placebo vs. alirocumab), the mean LDL-C reductions from baseline in patients receiving alirocumab 50, 100, and $150 \mathrm{mg}$ were $39.2,53.7$, and $61.0 \%$, respectively, versus placebo ( $\mathrm{p}<0.001$ for all). However, the duration of LDL$\mathrm{C}$ reduction was shorter in patients receiving atorvastatin, which may be a result of statin administration enhancing PCSK9 transcription and increasing circulating PCSK9 levels. Of note, alirocumab was generally well tolerated.

Subsequent phase II studies confirmed the promising results obtained with alirocumab in the aforementioned recycling of the LDL receptor to the plasma membrane, after binding the circulating LDL particle. The therapeutic approaches that inhibit circulating PCSK9 are highlighted in red boxes Reproduced with permission from Norata et al. [68]

phase I studies. A phase II study was carried out in 183 hypercholesterolemic patients with LDL-C $>100 \mathrm{mg} / \mathrm{dL}$ despite stable atorvastatin therapy [40] treated with subcutaneous alirocumab 50,100, or $150 \mathrm{mg}$ Q2W, or with 200 or $300 \mathrm{mg}$ every 4 weeks (Q4W), alternating with placebo for a total treatment period of 12 weeks. Reductions in LDL-C from baseline ranged from 40 to 72 and 43 to $48 \%$ in patients treated $\mathrm{Q} 2 \mathrm{~W}$ and $\mathrm{Q} 4 \mathrm{~W}$, respectively. In this study, alirocumab was generally well tolerated, but one patient experienced leukocytoclastic vasculitis. Although 
the exact cause of the leukocytoclastic vasculitis in this subject cannot be determined, the investigators considered it related to study drug administration [40]. Stein and coworkers reported the results of a phase II, randomized, multicentre, placebo controlled study in which 77 patients with FH treated with statins with or without ezetimibe and with LDL-C levels $>100 \mathrm{mg} / \mathrm{dL}$ underwent treatment with alirocumab 150,200 , or $300 \mathrm{mg}$ Q4W or alirocumab $150 \mathrm{mg}$ Q2W, or placebo Q2W [41]. LDL-C levels were reduced by $28.87,31.54$, and $42.53 \%$ in the alirocumab 150,200 , and $300 \mathrm{mg}$ Q4W groups, respectively, and by $67.90 \%$ in the alirocumab $150 \mathrm{mg}$ Q2W group (versus $10.65 \%$ in placebo recipients). While Q4W dosing achieved similar reductions to the $150 \mathrm{mg}$ Q2W dose at 2 weeks, the LDL-C reduction was not fully maintained over the full 4 week-period [41]. In a subsequent phase II study [42] 92 patients who had LDL-C levels of $\geq 100 \mathrm{mg} /$ $\mathrm{dL}$ after treatment with $10 \mathrm{mg}$ of atorvastatin for at least 7 weeks were randomly assigned to receive 8 weeks of treatment with atorvastatin $80 \mathrm{mg} /$ day plus subcutaneous alirocumab $150 \mathrm{mg}$ Q2W, atorvastatin $10 \mathrm{mg} /$ day plus subcutaneous alirocumab $150 \mathrm{mg}$ Q2W, or atorvastatin $80 \mathrm{mg} /$ day plus placebo Q2W. As expected, the addition of alirocumab to atorvastatin was more effective at reducing LDL-C levels at 8 weeks than simply increasing the dosage of atorvastatin ( 3 ).

The phase III clinical trial program for alirocumab is named "ODYSSEY", and comprises 14 global phase 3 trials, including more than 23,500 patients across more than 2000 study centres in more than 40 countries around the world (Fig. 4). These trials primarily focused on three groups of patients with uncontrolled hypercholesterolemia on existing therapies: those with heFH, those at high $\mathrm{CV}$ risk, and those with statin intolerance. The majority of patients enrolled in the ODYSSEY trials were at high CV risk and were receiving maximally tolerated doses of statins.

The results of the ODYSSEY ALTERNATIVE, ODYSSEY HIGH FH, ODYSSEY COMBO I and ODYSSEY OPTIONS I and II have been published [43-46]; ODYSSEY CHOICE I and II studies are only available as conference abstracts at the time of writing; results from these studies were presented at the International Symposium on Atherosclerosis in May 2015. ODYSSEY ALTERNATIVE enrolled 361 patients with documented statin intolerance, with LDL-C $\geq 70 \mathrm{mg} / \mathrm{dL}$ and very high $\mathrm{CV}$ risk or LDL-C $\geq 100 \mathrm{mg} / \mathrm{dL}$ and moderate/high CV risk; a single-blind subcutaneous and oral placebo was given to the patients for four weeks to check for placebo induced muscle-related adverse events. Patients reporting adverse events were withdrawn from the study and the others were randomized (2:2:1 ratio) to alirocumab $75 \mathrm{mg}$ self-administered via single $1 \mathrm{~mL}$ prefilled pen every 2 weeks or ezetimibe $10 \mathrm{mg} /$ day or atorvastatin $20 \mathrm{mg} /$ day (statin re-challenge), for 24 weeks. Patients received alirocumab $75 \mathrm{mg}$ Q2W with the possibility of uptitration to alirocumab $150 \mathrm{mg}$ Q2W at week 12 depending on $\mathrm{CV}$ risk and if LDL-C goals were not achieved by week 8 . The primary efficacy analysis showed that after 24 weeks, alirocumab treatment resulted in a significantly greater LDL-C reduction from baseline than ezetimibe treatment. Adverse events were generally similar between groups; skeletal muscle-related treatment-emergent adverse events occurred significantly less frequently in the alirocumab group versus the atorvastatin group $(p=0.042)$. ODYSSEY HIGH FH compared the LDL-Clowering efficacy and safety of subcutaneous alirocumab and placebo in heFH patients with LDL-C $\geq 160 \mathrm{mg} / \mathrm{dL}$ despite maximally tolerated statin with or without other lipid-lowering treatments. Alirocumab $150 \mathrm{mg}$ Q2W produced significantly greater LDL-C reductions from baseline versus placebo at week 24 , and had an excellent safety profile. In ODYSSEY COMBO I, 316 patients with hypercholesterolemia and documented CVD (established CHD or CHD risk equivalents) who were receiving maximally tolerated doses of statins with or without other lipidlowering therapies were randomised to receive either alirocumab or placebo; if patients had not achieved LDL-C goals by week 8 , there was an option to increase alirocumab to $150 \mathrm{mg}$ Q2W. Patients receiving alirocumab had significantly greater reductions from baseline in LDLC compared with placebo recipients $(\mathrm{p}<0.0001)$, while treatment-emergent adverse events were similar between groups. ODYSSEY OPTIONS I and II investigated the efficacy and safety of alirocumab as add-on therapy to atorvastatin versus ezetimibe plus atorvastatin, the doubling of the atorvastatin dose, or switching from atorvastatin to rosuvastatin in high $\mathrm{CV}$ risk patients with hypercholesterolemia who were not at goal despite existing therapy with non-maximal doses of atorvastatin. At 24 weeks, the alirocumab groups experienced greater LDL-C reductions compared with other treatment options; safety and tolerability was comparable across all groups.

The ODYSSEY CHOICE I study enrolled patients with hypercholesterolemia who had: a moderate to very high $\mathrm{CV}$ risk and were receiving maximally-tolerated statin doses; a moderate $\mathrm{CV}$ risk and were not receiving statins; or a moderate to very high CV risk and statin intolerance. Patients received either alirocumab $300 \mathrm{mg}$ Q4W, alirocumab $75 \mathrm{mg}$ Q2W, or placebo; patients who had not achieved LDL-C goals at week 8 received alirocumab $150 \mathrm{mg}$ Q2W from week 12. After 24 weeks of treatment, patients receiving alirocumab $300 \mathrm{mg}$ Q4W had significantly greater decreases from baseline in LDL-C versus placebo recipients, regardless of whether patients were receiving statin therapy $(\mathrm{p}<0.0001)$. Adverse events 


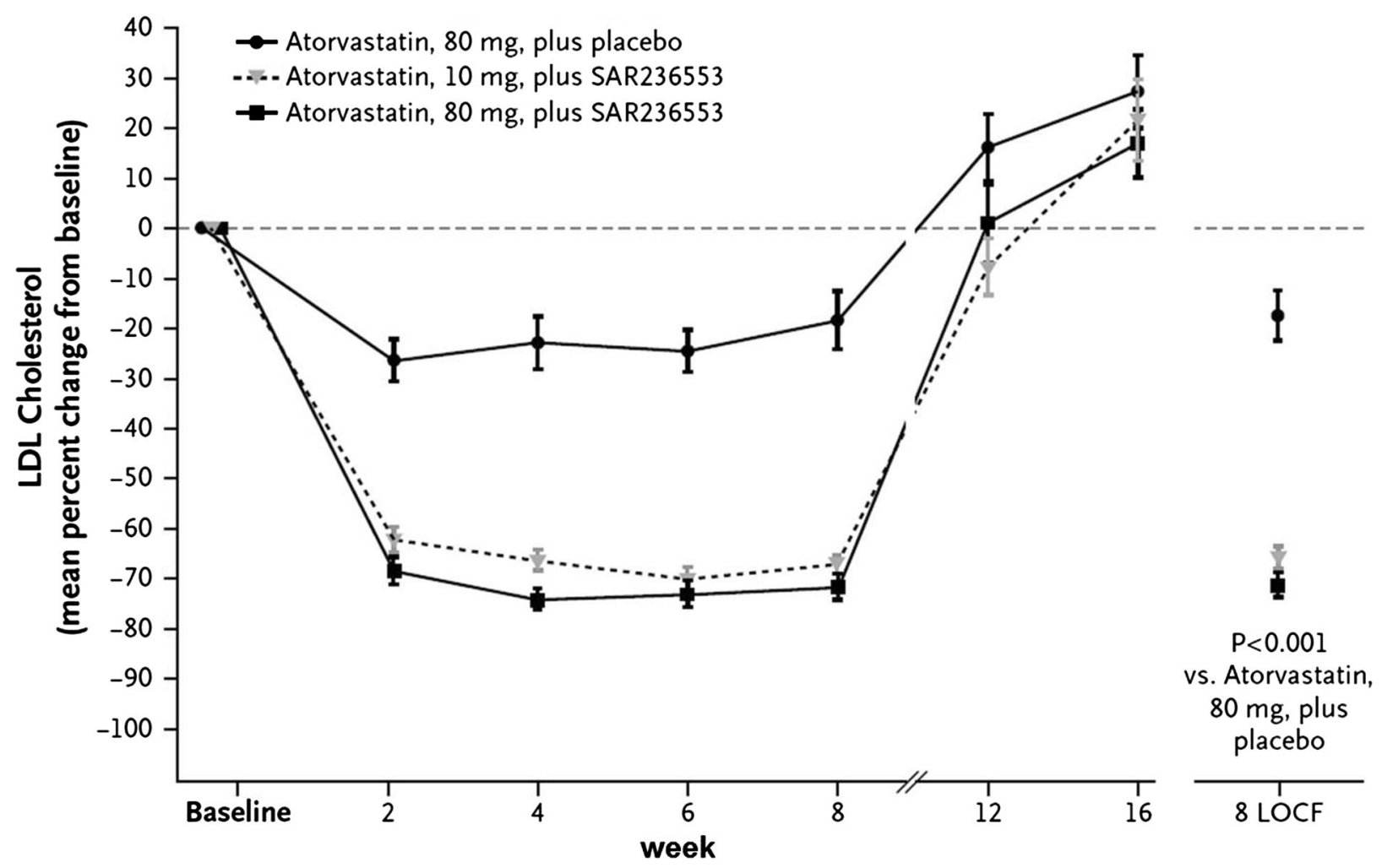

Fig. 3 Mean percent change from baseline in LDL-C levels in patients atorvastatin with or without alirocumab. Reproduced with permission from Roth et al. [42]

occurred at a similar rate in the placebo and alirocumab $300 \mathrm{mg}$ groups; there was a higher rate of injection site reactions in patients receiving alirocumab $300 \mathrm{mg}$ Q4W than placebo. The CHOICE II study included patients with hypercholesterolemia who were receiving fenofibrate, ezetimibe or diet alone and who had a moderate to very high CV risk plus SAMS, or a moderate CV risk without SAMS. Patients received either alirocumab $150 \mathrm{mg}$ Q4W, alirocumab $75 \mathrm{mg}$ Q2W, or placebo; patients who had not achieved LDL-C goals at week 8 , or whose LDL-C reduction was $<30 \%$ from baseline, received alirocumab $150 \mathrm{mg}$ Q2W from week 12. At week 24, alirocumab $150 \mathrm{mg}$ Q4W recipients had significantly greater LDL-C reductions from baseline than placebo recipients $(\mathrm{p}<0.0001)$.

The primary results of the ODYSSEY FH I and II, ODYSSEY MONO, ODYSSEY COMBO II, and ODYSSEY LONG TERM have been fully published [47-50]. In the ODYSSEY FH I and II studies, 735 heFH patients treated with a maximally tolerated statin dose with or without other lipid-lowering therapies with LDL-C $\geq 70 \mathrm{mg} / \mathrm{dL}$ (plus history of CVD) or $\geq 100 \mathrm{mg} / \mathrm{dL}$ (plus no history of CVD) were randomized to subcutaneous alirocumab $75 \mathrm{mg}$ Q2W or to placebo; the dose was increased to $150 \mathrm{mg}$ Q2W if the patient's LDL-C level remained $\geq 70 \mathrm{mg} / \mathrm{dL}$ at week 8 . Alirocumab produced significantly greater LDL-C reductions versus placebo at week 24 . Safety and tolerability were generally comparable in the alirocumab and placebo groups. ODYSSEY MONO investigated the use of alirocumab as monotherapy [49]; a total of 103 patients were randomly assigned to an initial dose of alirocumab $75 \mathrm{mg}$ Q2W (with the possibility of uptitration at 12 weeks if LDL-C level remained $\geq 70 \mathrm{mg} / \mathrm{dL}$ at week 8 ) or ezetimibe $10 \mathrm{mg}$ once daily. After 24 weeks, the alirocumab group showed a significantly greater least-squares mean reduction in LDL-C than ezetimibe $(47.2 \%$ vs. $15.6 \%$; p $<0.0001)$. Adverse events were similar between the two groups. ODYSSEY COMBO II compared the efficacy and safety of alirocumab versus ezetimibe in 720 patients with high $\mathrm{CV}$ risk and elevated LDL-C despite maximal doses of statins (LDL-C $\geq 70 \mathrm{mg} / \mathrm{dL}$ plus history of CVD, or LDL-C $\geq 100 \mathrm{mg} / \mathrm{dL}$ with no history of CVD) [47]. Patients were randomized to subcutaneous alirocumab $75 \mathrm{mg}$ Q2W (plus oral placebo) or oral ezetimibe $10 \mathrm{mg} /$ day (plus subcutaneous placebo) on a background of stable statin therapy, with an option to uptitrate alirocumab to $150 \mathrm{mg}$ Q2W at week 12 in patients who were not at goal at week 8 . The primary efficacy analysis showed that at 24 weeks, alirocumab recipients had a significantly greater reduction 


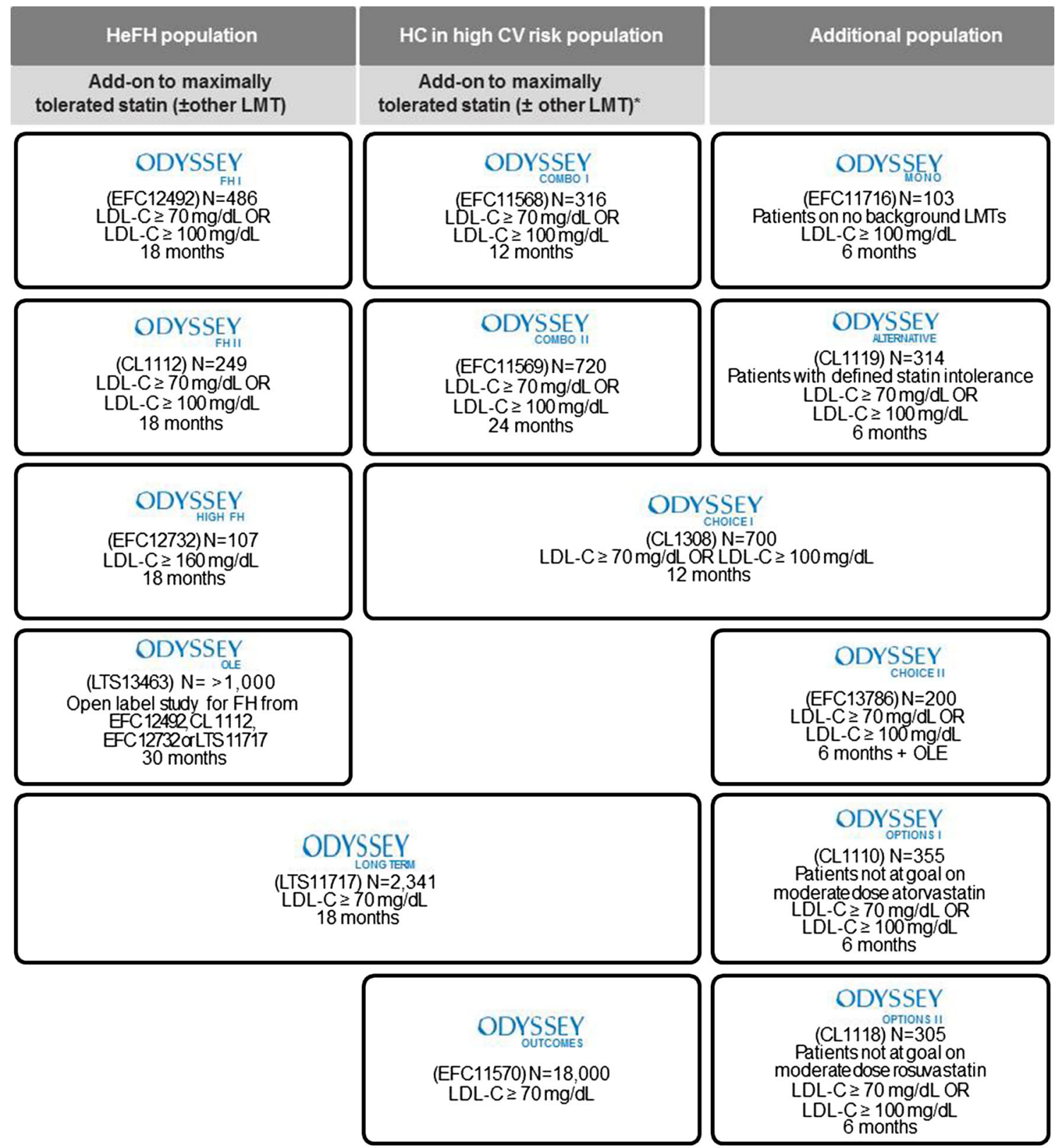

Fig. 4 The ODYSSEY clinical trial program. $C V$ cardiovascular, $F H$ familial hypercholesterolemia, $H C$ hypercholesterolemia, $\mathrm{HeFH}$ heterozygous familial hypercholesterolemia, $L D L-C$ low density

from baseline in LDL-C than ezetimibe recipients ( $p<0.0001$; Fig. 5). Alirocumab was generally well tolerated, with no evidence of an excess of treatment-emergent adverse events.

In the double-blind, randomized, placebo controlled ODYSSEY LONG TERM trial, 2341 patients with heterozygous $\mathrm{FH}$ with or without documented CVD or patients with hypercholesterolemia and documented CVD and LDL-C levels $\geq 70 \mathrm{mg} / \mathrm{dL}$ despite receiving the maximum tolerated doses of statins with or without other lipidlowering therapy, were randomly assigned subcutaneous lipoprotein cholesterol, LMT lipid modifying therapy. Asterisk For the ODYSSEY COMBO II other LMT not allowed at entry

alirocumab $150 \mathrm{mg}$ or placebo Q2W for 78 weeks. At week 24, the difference between the alirocumab and placebo groups in the mean percentage change from baseline in LDL-C levels was significant ( $p<0.001)$; the treatment effect remained consistent up to 78 weeks (Fig. 6). The percentage of patients with any adverse event was comparable between the two study groups. Interestingly, in a post-hoc analysis, the rate of major adverse CV events (death from coronary heart disease, nonfatal myocardial infarction, fatal or nonfatal ischemic stroke, or unstable angina requiring hospitalization) was lower with 


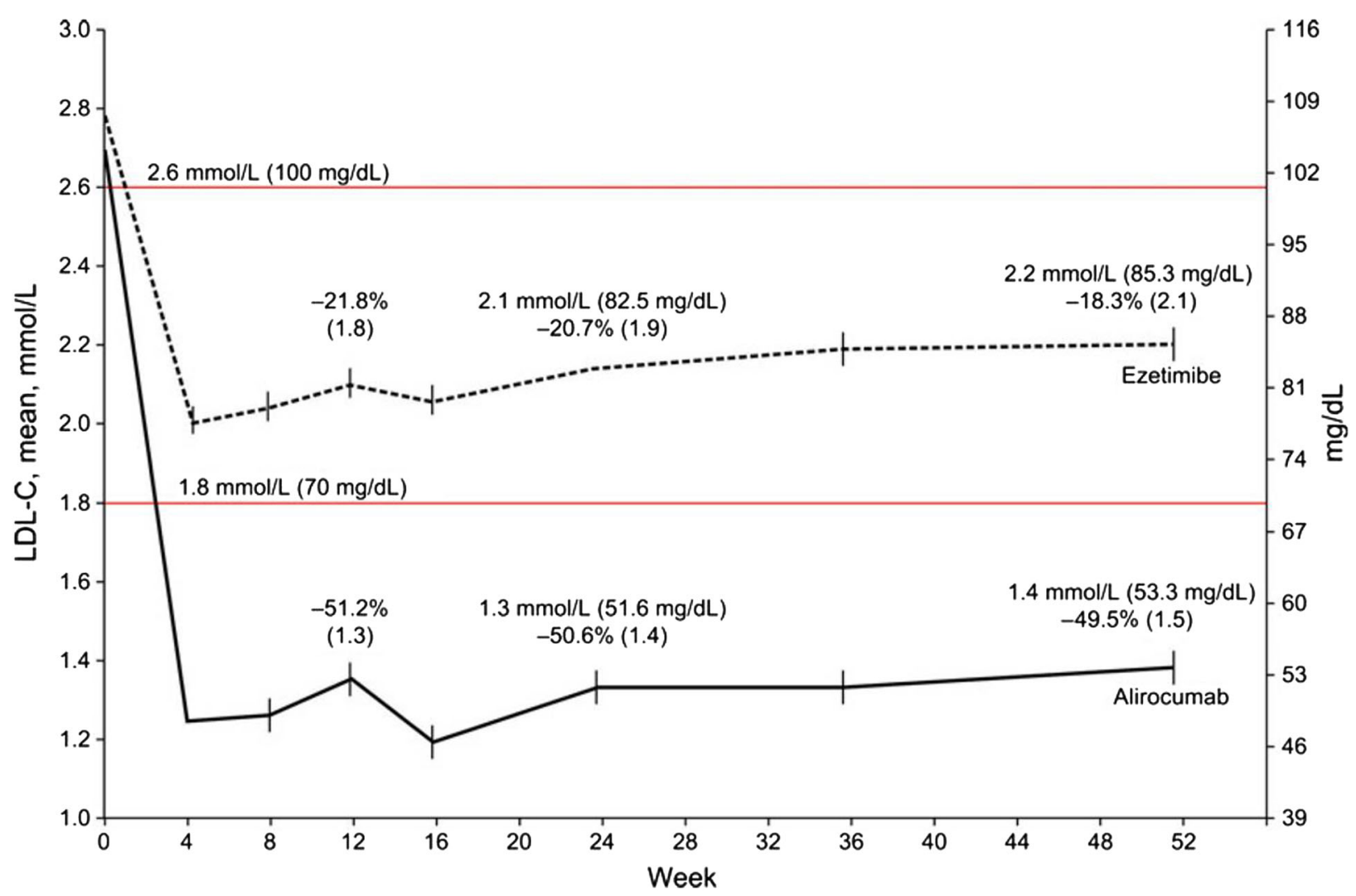

Fig. 5 Reduction in LDL-C levels over time in the intent-to-treat population of ODYSSEY COMBO. Reproduced with permission from Cannon et al. [47]

alirocumab than with placebo (1.7 vs. $3.3 \%$; HR: 0.52 ; $95 \%$ CI $0.31-0.90 ; \mathrm{p}=0.02)$.

Undoubtedly, the results of the ODYSSEY LONG TERM study are of great interest. The trial confirmed, in a large population with high CV risk, that treatment with alirocumab in patients already taking statins is well tolerated, and that it provides a reduction in LDL-C of more than $60 \%$, which might translate into a $50 \%$ decrease in $\mathrm{CV}$ events. Of course, this last finding requires confirmation, in view of the relatively small number of $\mathrm{CV}$ events observed in the trial, the post hoc nature of the analysis, and the relatively short duration of the observation period. However, the ODYSSEY results as a whole give some insights regarding the possible use of alirocumab not only in patients with $\mathrm{FH}$, but also in the large proportion of hypercholesterolemic patients at high $\mathrm{CV}$ risk that fail to reach appropriate lipid targets on statin therapy.

This issue is being formally tested by the ongoing phase III ODYSSEY Outcomes trial (ClinicalTrials.gov number: NCT01663402), that will test the hypothesis that alirocumab, compared with subcutaneous placebo, reduces $\mathrm{CV}$ morbidity and mortality in patients with recent acute coronary syndrome and lipid levels that remain above target despite intensive atorvastatin or rosuvastatin therapy (or the maximally tolerated dose) [51]. Planned enrolment is 18,000 patients who will be randomized to receive alirocumab $75 \mathrm{mg}$ Q2W, by subcutaneous injection or matching placebo, with the option to uptitrate to alirocumab $150 \mathrm{mg}$ Q2W after 1 month, if LDL-C levels remain $\geq 50 \mathrm{mg} / \mathrm{dL}$; the protocol also includes the option to downtitrate from alirocumab $150 \mathrm{mg}$ Q2W to $75 \mathrm{mg} \mathrm{Q} 2 \mathrm{~W}$ in patients who have two consecutive LDL-C levels of $<25 \mathrm{mg} / \mathrm{dL}$. The primary efficacy endpoint is the composite of: time to first occurrence of death from coronary heart disease; nonfatal acute myocardial infarction; fatal or nonfatal ischemic stroke; or unstable angina requiring hospitalization. The trial will continue until 1613 primary end point events have occurred; follow up is expected to be a minimum of 2 years for each subject.

\subsection{Evolocumab}

Evolocumab is a monoclonal antibody against PCSK9 which is approved for use in the US as an adjunct to diet and maximally tolerated statin therapy for the treatment of adults with $\mathrm{HeFH}$, homozygous $\mathrm{FH}(\mathrm{HoFH})$ or clinical atherosclerotic CVD, who require additional lowering of LDL-C [52]. In Europe, evolocumab is approved for the 


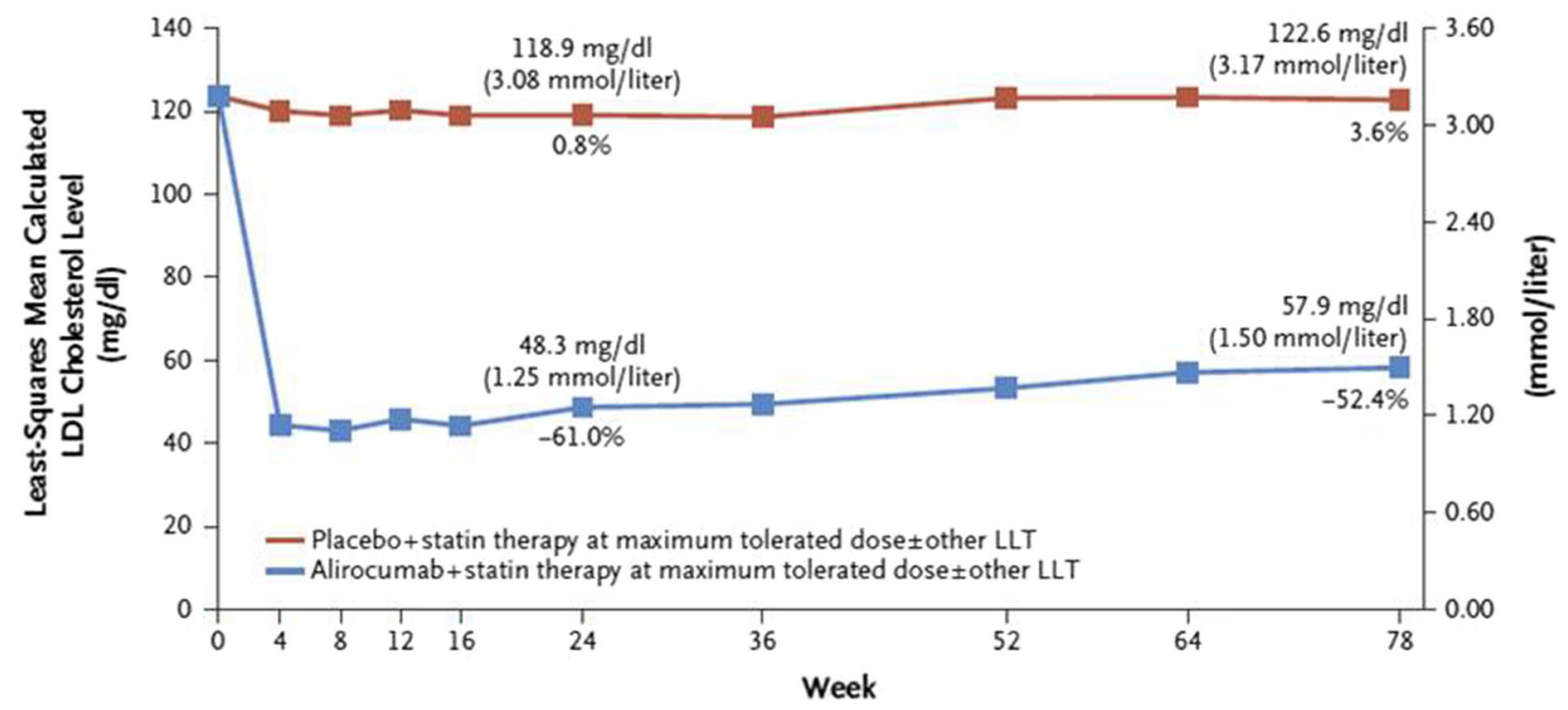

\section{No. of Patients with Data Available} Placebo $\begin{array}{lllllll}780 & 754 & 747 & 746 & 716 & 708 & 694\end{array}$ Alirocumab $\quad 15301473145814361412 \quad 1386$

Fig. 6 Reduction in LDL-C levels over time in the intent-to-treat population of ODYSSEY LONG TERM. Reproduced with permission from Robinson et al. [48]

treatment of adults with primary hypercholesterolemia (heterozygous familial and non-familial) or mixed dyslipidemia, as an adjunct to diet either in combination with a statin or a statin with other lipid lowering therapies in patients unable to reach LDL-C goals with the maximum tolerated dose of a statin, or alone or in combination with other lipid-lowering therapies in patients who are statinintolerant, or those for whom a statin is contraindicated. It is also indicated in adults and adolescents aged 12 years and over with $\mathrm{HoFH}$ in combination with other lipid-lowering therapies [53]. After the positive results of the phase I studies, in which the subcutaneous administration of evolocumab led to consistent reductions in LDL-C, subsequent phase II studies further confirmed the efficacy and safety of the compound. The phase II LAPLACE-TIMI 57 study [54] tested multiple doses of the drug $(70,105$ or $140 \mathrm{mg}$ Q2W and 280, 350 or $420 \mathrm{mg}$ Q4 W) versus matching placebo administered Q2W or Q4W in 631 patients already on treatment with a statin with or without ezetimibe and with LDL-C $\geq 85 \mathrm{mg} / \mathrm{dL}$. At week 12, mean LDL-C concentrations were reduced in a dose-dependent manner by evolocumab Q2W (41.8-66.1\%; $\mathrm{p}<0.0001$ vs. placebo) and evolocumab Q4W (41.8-50.3\%; $\mathrm{p}<0.0001$ vs. placebo). Treatment-related adverse events were similar in the evolocumab and placebo groups (8 vs $7 \%$ ) and none were severe. The efficacy and safety of the compound were confirmed by the results of the MENDEL study, which compared evolocumab with ezetimibe or placebo in 406 patients with hypercholesterolemia not receiving concurrent lipid-lowering treatment [55]. Similarly, the drug led to significant reductions in lipid levels in the RUTHERFORD study, in 167 patients with heFH with LDL-C $\geq 100 \mathrm{mg} / \mathrm{dL}$ despite statin therapy with or without ezetimibe [56], and in the GAUSS study, in 160 statin intolerant patients (defined as those with an inability to tolerate $\geq 1$ statin at any dose, or a dose above weekly maximums of statins due to intolerable myalgia or myopathy, who have an improvement in or resolution of symptoms when statins are discontinued) [57]. These studies confirmed a favourable safety profile of the drug.

A number of phase III trials have investigated or are investigating the efficacy and safety profile of evolocumab in various subsets of patients. In the LAPLACE- 2 trial, the compound was effective and safe when administered with either moderate- or high-intensity statin therapy [58], while in RUTHERFORD-2, in patients with heFH and LDL-C level $\geq 100 \mathrm{mg} / \mathrm{dL}$ despite statin therapy, the drug proved to be safe and efficacious in lowering lipid levels [59]. Three phase III trials, MENDEL 2, GAUSS 2 and DESCARTES 2, have been published in 2014, and demonstrated consistent reductions in LDL-C in different subsets of patients, including patients with Framingham risk scores $\leq 10 \%$ and LDL-C levels $\geq 100$ and $<190 \mathrm{mg} / \mathrm{dL}$ [60], patients with statin intolerance [61], and in patients with 
LDL-C level $>75 \mathrm{mg} / \mathrm{dL}$ despite lipid-lowering therapy with atorvastatin with or without ezetimibe [62]. In these three studies evolocumab led to a reduction in LDL-C from baseline of between 50 and $60 \%$.

The OSLER-1 and OSLER-2 open-label trials included 4465 patients with various degrees of CV risk who were randomly assigned to receive either evolocumab $140 \mathrm{mg}$ Q2W or $420 \mathrm{mg}$ Q4W plus standard therapy, or standard therapy alone [63]. Versus standard therapy alone, evolocumab reduced LDL-C levels by $61 \%$ (95\% CI 59-63; $\mathrm{p}<0.001$ ), and this reduction was maintained at 48 weeks $(58.4 \% ; \mathrm{p}<0.001)$. The rate of adverse events was low, with no difference between groups. Interestingly, an exploratory analysis showed that the rate of $\mathrm{CV}$ events at 1 year was reduced with evolocumab versus standard therapy [0.95 vs. $2.18 \%$; HR (evolocumab group): 0.47 ; $95 \%$ CI $0.28-0.78 ; \mathrm{p}=0.003]$. These interesting results require confirmation in larger studies specifically designed for such endpoints, such as the ongoing FOURIER study (ClinicalTrials.gov number: NCT01764633), which is a randomized, placebo-controlled trial currently recruiting a planned 27,500 high-risk patients with CVD receiving background statin therapy which will test the effect of evolocumab versus placebo on major CVD events.

\subsection{Bococizumab}

Bococizumab is a humanized monoclonal antibody against PCSK9 which is in an earlier phase of development compared with alirocumab and evolocumab. The results of phase II studies have been presented in abstract form and seem to indicate that the compound is effective and generally well tolerated. In two studies presented at the AHA Scientific Sessions in 2012, substantial LDL-C lowering was observed in hypercholesterolemic subjects treated with the compound (single intravenous and subcutaneous doses), both as monotherapy and when added to atorvastatin therapy. The drug was well-tolerated.

To date the only phase II study published in extenso is a dose ranging trial [64] in which subjects with LDL-C levels $\geq 80 \mathrm{mg} / \mathrm{dL}$ on stable statin therapy were randomized to Q14 days of subcutaneous placebo or bococizumab 50, 100 , or $150 \mathrm{mg}$ or Q28 days of subcutaneous placebo or bococizumab 200 or $300 \mathrm{mg}$. When results were adjusted for placebo, mean decreases from baseline in LDL-C ranged from 35.0 to $53.1 \%$ in patients receiving bococizumab for 14 days, and from 27.0 to $41.1 \%$ in patients receiving 28 days of bococizumab therapy. As observed in trials with other PCSKS9 monoclonal antibodies, the percentage of subjects reporting adverse events or serious adverse events was similar across placebo and bococizumab treatment groups; only 7 subjects (2\%) discontinued treatment because of treatment-related adverse events.
The ongoing SPIRE program, which includes 5 phase III trials, will further evaluate the efficacy and safety of bococizumab in various subsets of patients; the results of these studies are expected in 2016 and 2017.

\subsection{Lomitapide}

Lomitapide inhibits the microsomal triglyceride transport protein (MTP), a key protein in the assembly and secretion of apoB-containing lipoproteins in the liver and intestine [65]. Inhibiting MTP leads to reduced levels of these lipoproteins in the circulation. The efficacy and safety of oral lomitapide has been evaluated in three small clinical studies [65, 66]. The drug was approved in 2012 by the US FDA and in 2013 by the European Medicines Agency for patients with homozygous FH. The possible relevant liver toxicity seen represents the main limitation of the drug; also other gastrointestinal side effects have been described (diarrhoea, nausea and vomiting) [65, 66].

\subsection{Mipomersen}

Mipomersen is an antisense oligonucleotide complementary to the coding region for human apolipoprotein B messenger RNA (mRNA) [65]. Through direct binding to apoB mRNA, mipomersen inhibits its production. The effectiveness of mipomersen $400 \mathrm{mg}$ administered subcutaneously once weekly has been tested in relatively small studies in which the reduction in LDL-C ranged between 25 and $40 \%$ [65, 67]. In these studies, the most common adverse events have been injection site reactions (76 \% of patients), flu-like symptoms ( $\sim 30 \%$, typically appearing 2 days after injection), and persistent increases in serum alanine aminotransferase levels more than three times the upper limit of normal ( $\sim 10 \%)[65,67]$.

A field of ongoing research is that which is aiming to increase HDL-C by increasing the activity of apolipoprotein A-I. These include infusions of recombinant HDL-C or apolipoprotein A-I Milano, mimetic peptides and oral small molecules [11].

\section{Conclusion}

Control of lipid levels is one of the most effective strategies for CVD prevention. More than 25 years of clinical trial data have clearly demonstrated that LDL-C lowering, primarily with statins, reduces major $\mathrm{CV}$ events and mortality. However, data from clinical trials and surveys clearly indicate that a large proportion of patients fail to achieve lipid goals, and this is frequent in patients at high or very high CV risk, possibly leading to a high economic and social burden. 
The development of novel lipid-lowering agents with different mechanisms of action versus existing therapies represents an appealing option for the treatment of patients who fail to achieve lipid targets. In particular, a few years after the discovery of rare PCSK9 mutations that may strongly influence lipid profile and affect CV risk, PCSK9 inhibitors are being recognized as promising therapeutic options for the treatment of hypercholesterolemia, in view of their tolerability and efficacy in lowering LDL-C and data suggesting a possible positive trend in $\mathrm{CV}$ outcomes with the use of PCKS9 inhibitors in the treatment of hypercholesterolemia. If the promising results obtained so far with monoclonal antibodies against PCKS9 are confirmed by the ongoing large phase III outcomes studies, clinicians will be provided with a new powerful tool for lipid-level reduction and an optimal way to control CV risk.

Acknowledgments We thank Sheridan Henness, $\mathrm{PhD}$, of Springer Healthcare Communications, for technical and English editing assistance, which was funded by Sanofi Aventis, Italy.

\section{Compliance with Ethical Standards}

Conflict of interest EAR has received speaker honoraria from Amgen and Sanofi; MS declares to have no Conflicts of Interest.

Ethical statement This article does not contain any studies with human participants or animals performed by any of the authors.

Open Access This article is distributed under the terms of the Creative Commons Attribution-NonCommercial 4.0 International License (http://creativecommons.org/licenses/by-nc/4.0/), which permits any noncommercial use, distribution, and reproduction in any medium, provided you give appropriate credit to the original author(s) and the source, provide a link to the Creative Commons license, and indicate if changes were made.

\section{References}

1. Perk J, De Backer G, Gohlke H, Graham I, Reiner Z, Verschuren M, Albus C, et al. European Guidelines on cardiovascular disease prevention in clinical practice (version 2012): the Fifth Joint Task Force of the European Society of Cardiology and Other Societies on Cardiovascular Disease Prevention in Clinical Practice (constituted by representatives of nine societies and by invited experts). Eur J Prev Cardiol. 2012;19:585-667.

2. World Health Organization (2014) Global status report on noncommunicable diseases. http://apps.who.int/iris/bitstream/10665/ 148114/1/9789241564854_eng.pdf?ua=1. Accessed 5 Feb 2016.

3. Tunstall-Pedoe $\mathrm{H}$, Kuulasmaa $\mathrm{K}$, Tolonen $\mathrm{H}$, Davidson $\mathrm{M}$, Mendis S, the WHO MONICA project team (2003) MONICA monograph and multimedia source book.

4. Verschuren WM, Jacobs DR, Bloemberg BP, Kromhout D, Menotti A, Aravanis C, Blackburn H, et al. Serum total cholesterol and long-term coronary heart disease mortality in different cultures. Twenty-five-year follow-up of the seven countries study. JAMA. 1995;274:131-6.
5. Tomkin GH, Owens D. LDL as a cause of atherosclerosis. Open Atheroscler Thromb J. 2012;5:13-21.

6. Anderson TJ, Gregoire J, Hegele RA, Couture P, Mancini GB, McPherson R, Francis GA, et al. 2012 update of the Canadian Cardiovascular Society guidelines for the diagnosis and treatment of dyslipidemia for the prevention of cardiovascular disease in the adult. Can J Cardiol. 2013;29:151-67.

7. Stone NJ, Robinson JG, Lichtenstein AH, Bairey Merz CN, Blum CB, Eckel RH, Goldberg AC, et al. 2013 ACC/AHA guideline on the treatment of blood cholesterol to reduce atherosclerotic cardiovascular risk in adults: a report of the American College of Cardiology/American Heart Association Task Force on Practice Guidelines. Circulation. 2014;129:S1-45.

8. American Diabetes Association. (8) Cardiovascular disease and risk management. Diabetes Care. 2015;38(Suppl):S49-57.

9. Reiner Z, Catapano AL, De Backer G, Graham I, Taskinen MR, Wiklund O, Agewall S, et al. ESC/EAS Guidelines for the management of dyslipidaemias: the Task Force for the management of dyslipidaemias of the European Society of Cardiology (ESC) and the European Atherosclerosis Society (EAS). Eur Heart J. 2011;32:1769-818.

10. Baigent C, Blackwell L, Emberson J, Holland LE, Reith C, Bhala $\mathrm{N}$, Peto R, et al. Efficacy and safety of more intensive lowering of LDL cholesterol: a meta-analysis of data from 170,000 participants in 26 randomised trials. Lancet. 2010;376:1670-81.

11. Gotto AM Jr, Moon JE. Twenty-five years of statins: where do we go from here? Clin Lipidol. 2015;10:33-45.

12. Cannon CP, Blazing MA, Giugliano RP, McCagg A, White JA, Theroux P, Darius H, et al. Ezetimibe Added to Statin Therapy after Acute Coronary Syndromes. N Engl J Med. 2015;372:2387-97.

13. Boekholdt SM, Hovingh GK, Mora S, Arsenault BJ, Amarenco P, Pedersen TR, LaRosa JC, et al. Very low levels of atherogenic lipoproteins and the risk for cardiovascular events: a meta-analysis of statin trials. J Am Coll Cardiol. 2014;64:485-94.

14. Kotseva K, Wood D, De Bacquer D, De Backer G, Ryden L, Jennings C, Gyberg V, et al. EUROASPIRE IV: a European Society of Cardiology survey on the lifestyle, risk factor and therapeutic management of coronary patients from 24 European countries. Eur J Prev Cardiol. 2016;23:636-48.

15. De Smedt D, Kotseva K, De Bacquer D, Wood D, De Backer G, Dallongeville J, Seppo L, et al. Cost-effectiveness of optimizing prevention in patients with coronary heart disease: the EUROASPIRE III health economics project. Eur Heart J. 2012;33:2865-72.

16. Mehta RH, Bhatt DL, Steg PG, Goto S, Hirsch AT, Liau CS, Rother J, et al. Modifiable risk factors control and its relationship with 1 year outcomes after coronary artery bypass surgery: insights from the REACH registry. Eur Heart J. 2008;29:3052-60.

17. Teo K, Lear S, Islam S, Mony P, Dehghan M, Li W, Rosengren $\mathrm{A}$, et al. Prevalence of a healthy lifestyle among individuals with cardiovascular disease in high-, middle- and low-income countries: the prospective urban rural epidemiology (PURE) study. JAMA. 2013;309:1613-21.

18. Naoumova RP, Tosi I, Patel D, Neuwirth C, Horswell SD, Marais $\mathrm{AD}$, van Heyningen C, et al. Severe hypercholesterolemia in four British families with the D374Y mutation in the PCSK9 gene: long-term follow-up and treatment response. Arterioscler Thromb Vasc Biol. 2005;25:2654-60.

19. Timms KM, Wagner S, Samuels ME, Forbey K, Goldfine H, Jammulapati S, Skolnick MH, et al. A mutation in PCSK9 causing autosomal-dominant hypercholesterolemia in a Utah pedigree. Hum Genet. 2004;114:349-53.

20. Pfizer Atorvastatin: summary of product characteristics.

21. AstraZeneca Rosuvastatin: summary of product characteristics. 
22. Pijlman AH, Huijgen R, Verhagen SN, Imholz BP, Liem AH, Kastelein JJ, Abbink EJ, et al. Evaluation of cholesterol lowering treatment of patients with familial hypercholesterolemia: a large cross-sectional study in The Netherlands. Atherosclerosis. 2010;209:189-94.

23. Senior V, Marteau TM, Weinman J. Self-reported adherence to cholesterol-lowering medication in patients with familial hypercholesterolaemia: the role of illness perceptions. Cardiovasc Drugs Ther. 2004;18:475-81.

24. Corrao G, Conti V, Merlino L, Catapano AL, Mancia G. Results of a retrospective database analysis of adherence to statin therapy and risk of nonfatal ischemic heart disease in daily clinical practice in Italy. Clin Ther. 2010;32:300-10.

25. Liberopoulos EN, Florentin M, Mikhailidis DP, Elisaf MS. Compliance with lipid-lowering therapy and its impact on cardiovascular morbidity and mortality. Expert Opin Drug Saf. 2008;7:717-25.

26. Stroes ES, Thompson PD, Corsini A, Vladutiu GD, Raal FJ, Ray KK, Roden M, et al. Statin-associated muscle symptoms: impact on statin therapy-European Atherosclerosis Society Consensus Panel Statement on Assessment, Aetiology and Management. Eur Heart J. 2015;36:1012-22.

27. Bruckert E, Hayem G, Dejager S, Yau C, Begaud B. Mild to moderate muscular symptoms with high-dosage statin therapy in hyperlipidemic patients-the PRIMO study. Cardiovasc Drugs Ther. 2005;19:403-14.

28. Buettner C, Rippberger MJ, Smith JK, Leveille SG, Davis RB, Mittleman MA. Statin use and musculoskeletal pain among adults with and without arthritis. Am J Med. 2012;125:176-82.

29. Cohen JD, Brinton EA, Ito MK, Jacobson TA. Understanding Statin Use in America and Gaps in Patient Education (USAGE): an internet-based survey of 10,138 current and former statin users. J Clin Lipidol. 2012;6:208-15.

30. El-Salem K, Ababneh B, Rudnicki S, Malkawi A, Alrefai A, Khader $\mathrm{Y}$, Saadeh R, et al. Prevalence and risk factors of muscle complications secondary to statins. Muscle Nerve. 2011;44:877-81.

31. Zhang H, Plutzky J, Skentzos S, Morrison F, Mar P, Shubina M, Turchin A. Discontinuation of statins in routine care settings: a cohort study. Ann Intern Med. 2013;158:526-34.

32. Norata GD, Ballantyne CM, Catapano AL. New therapeutic principles in dyslipidaemia: focus on LDL and Lp(a) lowering drugs. Eur Heart J. 2013;34:1783-9.

33. Seidah NG, Benjannet S, Wickham L, Marcinkiewicz J, Jasmin $\mathrm{SB}$, Stifani S, Basak A, et al. The secretory proprotein convertase neural apoptosis-regulated convertase 1 (NARC-1): liver regeneration and neuronal differentiation. Proc Natl Acad Sci USA. 2003;100:928-33.

34. Abifadel M, Varret M, Rabes JP, Allard D, Ouguerram K, Devillers M, Cruaud C, et al. Mutations in PCSK9 cause autosomal dominant hypercholesterolemia. Nat Genet. 2003;34:154-6.

35. Leren TP. Mutations in the PCSK 9 gene in Norwegian subjects with autosomal dominant hypercholesterolemia. Clin Genet. 2004;65:419-22.

36. Davignon J, Dubuc G, Seidah NG. The influence of PCSK9 polymorphisms on serum low-density lipoprotein cholesterol and risk of atherosclerosis. Curr Atheroscler Rep. 2010;12:308-15.

37. Cohen JC, Boerwinkle E, Mosley TH Jr, Hobbs HH. Sequence variations in PCSK9, low LDL, and protection against coronary heart disease. N Engl J Med. 2006;354:1264-72.

38. Benn M, Nordestgaard BG, Grande P, Schnohr P, TybjaergHansen A. PCSK9 R46L, low-density lipoprotein cholesterol levels, and risk of ischemic heart disease: 3 independent studies and meta-analyses. J Am Coll Cardiol. 2010;55:2833-42.

39. Stein EA, Mellis S, Yancopoulos GD, Stahl N, Logan D, Smith WB, Lisbon E, et al. Effect of a monoclonal antibody to PCSK9 on LDL cholesterol. N Engl J Med. 2012;366:1108-18.
40. McKenney JM, Koren MJ, Kereiakes DJ, Hanotin C, Ferrand AC, Stein EA. Safety and efficacy of a monoclonal antibody to proprotein convertase subtilisin/kexin type 9 serine protease, SAR236553/REGN727, in patients with primary hypercholesterolemia receiving ongoing stable atorvastatin therapy. J Am Coll Cardiol. 2012;59:2344-53.

41. Stein EA, Gipe D, Bergeron J, Gaudet D, Weiss R, Dufour R, Wu $\mathrm{R}$, et al. Effect of a monoclonal antibody to PCSK9, REGN727/ SAR236553, to reduce low-density lipoprotein cholesterol in patients with heterozygous familial hypercholesterolaemia on stable statin dose with or without ezetimibe therapy: a phase 2 randomised controlled trial. Lancet. 2012;380:29-36.

42. Roth EM, McKenney JM, Hanotin C, Asset G, Stein EA. Atorvastatin with or without an antibody to PCSK9 in primary hypercholesterolemia. N Engl J Med. 2012;367:1891-900.

43. Kastelein JJ, Robinson JG, Farnier M, Krempf M, Langslet G, Lorenzato C, Gipe DA, et al. Efficacy and safety of alirocumab in patients with heterozygous familial hypercholesterolemia not adequately controlled with current lipid-lowering therapy: design and rationale of the ODYSSEY FH studies. Cardiovasc Drugs Ther. 2014;28:281-9.

44. Moriarty PM, Jacobson TA, Bruckert E, Thompson PD, Guyton JR, Baccara-Dinet MT, Gipe D. Efficacy and safety of alirocumab, a monoclonal antibody to PCSK9, in statin-intolerant patients: design and rationale of ODYSSEY ALTERNATIVE, a randomized phase 3 trial. J Clin Lipidol. 2014;8:554-61.

45. Kereiakes DJ, Robinson JG, Cannon CP, Lorenzato C, Pordy R, Chaudhari U, Colhoun HM. Efficacy and safety of the proprotein convertase subtilisin/kexin type 9 inhibitor alirocumab among high cardiovascular risk patients on maximally tolerated statin therapy: The ODYSSEY COMBO I study. Am Heart J. 2015;169(906-915):e913.

46. Bays H, Gaudet D, Weiss R, Ruiz JL, Watts GF, Gouni-Berthold I, Robinson J, et al. Alirocumab as add-on to atorvastatin versus other lipid treatment strategies: ODYSSEY OPTIONS I randomized trial. J Clin Endocrinol Metab. 2015;100:3140-8.

47. Cannon CP, Cariou B, Blom D, McKenney JM, Lorenzato C, Pordy R, Chaudhari U, et al. Efficacy and safety of alirocumab in high cardiovascular risk patients with inadequately controlled hypercholesterolaemia on maximally tolerated doses of statins: the ODYSSEY COMBO II randomized controlled trial. Eur Heart J. 2015;36:1186-94.

48. Robinson JG, Farnier M, Krempf M, Bergeron J, Luc G, Averna M, Stroes ES, et al. Efficacy and safety of alirocumab in reducing lipids and cardiovascular events. N Engl J Med. 2015;372: 1489-99.

49. Roth EM, McKenney JM. ODYSSEY MONO: effect of alirocumab $75 \mathrm{mg}$ subcutaneously every 2 weeks as monotherapy versus ezetimibe over 24 weeks. Future Cardiol. 2015;11:27-37.

50. Kastelein JJ, Ginsberg HN, Langslet G, Hovingh GK, Ceska R, Dufour R, Blom D, et al. ODYSSEY FH I and FH II: 78 week results with alirocumab treatment in 735 patients with heterozygous familial hypercholesterolaemia. Eur Heart J. 2015;36: 2996-3003.

51. Schwartz GG, Bessac L, Berdan LG, Bhatt DL, Bittner V, Diaz R, Goodman SG, et al. Effect of alirocumab, a monoclonal antibody to PCSK9, on long-term cardiovascular outcomes following acute coronary syndromes: rationale and design of the ODYSSEY outcomes trial. Am Heart J. 2014;168:682-9.

52. Amgen (2015) Evolocumab: prescribing information.

53. Amgen Evolocumab: summary of product characteristics.

54. Giugliano RP, Desai NR, Kohli P, Rogers WJ, Somaratne R, Huang F, Liu T, et al. Efficacy, safety, and tolerability of a monoclonal antibody to proprotein convertase subtilisin/kexin type 9 in combination with a statin in patients with hypercholesterolaemia (LAPLACE-TIMI 57): a randomised, placebo- 
controlled, dose-ranging, phase 2 study. Lancet. 2012;380:2007-17.

55. Koren MJ, Scott R, Kim JB, Knusel B, Liu T, Lei L, Bolognese $\mathrm{M}$, et al. Efficacy, safety, and tolerability of a monoclonal antibody to proprotein convertase subtilisin/kexin type 9 as monotherapy in patients with hypercholesterolaemia (MENDEL): a randomised, double-blind, placebo-controlled, phase 2 study. Lancet. 2012;380:1995-2006.

56. Raal F, Scott R, Somaratne R, Bridges I, Li G, Wasserman SM, Stein EA. Low-density lipoprotein cholesterol-lowering effects of AMG 145, a monoclonal antibody to proprotein convertase subtilisin/kexin type 9 serine protease in patients with heterozygous familial hypercholesterolemia: the Reduction of LDL-C with PCSK9 Inhibition in Heterozygous Familial Hypercholesterolemia Disorder (RUTHERFORD) randomized trial. Circulation. 2012;126:2408-17.

57. Sullivan D, Olsson AG, Scott R, Kim JB, Xue A, Gebski V, Wasserman SM, et al. Effect of a monoclonal antibody to PCSK9 on low-density lipoprotein cholesterol levels in statin-intolerant patients: the GAUSS randomized trial. JAMA. 2012;308:2497-506.

58. Robinson JG, Nedergaard BS, Rogers WJ, Fialkow J, Neutel JM, Ramstad D, Somaratne R, et al. Effect of evolocumab or ezetimibe added to moderate- or high-intensity statin therapy on LDL-C lowering in patients with hypercholesterolemia: the LAPLACE-2 randomized clinical trial. JAMA. 2014;311:1870-82.

59. Raal FJ, Stein EA, Dufour R, Turner T, Civeira F, Burgess L, Langslet G, et al. PCSK9 inhibition with evolocumab (AMG 145) in heterozygous familial hypercholesterolaemia (RUTHERFORD-2): a randomised, double-blind, placebo-controlled trial. Lancet. 2015;385:331-40.

60. Koren MJ, Lundqvist P, Bolognese M, Neutel JM, Monsalvo ML, Yang J, Kim JB, et al. Anti-PCSK9 monotherapy for hypercholesterolemia: the MENDEL-2 randomized, controlled phase III clinical trial of evolocumab. J Am Coll Cardiol. 2014;63:2531-40.

61. Stroes E, Colquhoun D, Sullivan D, Civeira F, Rosenson RS, Watts GF, Bruckert E, et al. Anti-PCSK9 antibody effectively lowers cholesterol in patients with statin intolerance: the GAUSS2 randomized, placebo-controlled phase 3 clinical trial of evolocumab. J Am Coll Cardiol. 2014;63:2541-8.

62. Blom DJ, Hala T, Bolognese M, Lillestol MJ, Toth PD, Burgess L, Ceska R, et al. A 52-week placebo-controlled trial of evolocumab in hyperlipidemia. N Engl J Med. 2014;370:1809-19.

63. Sabatine MS, Giugliano RP, Wiviott SD, Raal FJ, Blom DJ, Robinson J, Ballantyne CM, et al. Efficacy and safety of evolocumab in reducing lipids and cardiovascular events. $\mathrm{N}$ Engl $\mathrm{J}$ Med. 2015;372:1500-9.

64. Ballantyne CM, Neutel J, Cropp A, Duggan W, Wang EQ, Plowchalk D, Sweeney K, et al. Results of bococizumab, a monoclonal antibody against proprotein convertase subtilisin/ kexin type 9, from a randomized, placebo-controlled, doseranging study in statin-treated subjects with hypercholesterolemia. Am J Cardiol. 2015;115:1212-21.

65. Rader DJ, Kastelein JJ. Lomitapide and mipomersen: two first-inclass drugs for reducing low-density lipoprotein cholesterol in patients with homozygous familial hypercholesterolemia. Circulation. 2014;129:1022-32.

66. Cuchel M, Blom DJ, Averna MR. Clinical experience of lomitapide therapy in patients with homozygous familial hypercholesterolaemia. Atheroscler Suppl. 2014;15:33-45.

67. Thomas GS, Cromwell WC, Ali S, Chin W, Flaim JD, Davidson M. Mipomersen, an apolipoprotein B synthesis inhibitor, reduces atherogenic lipoproteins in patients with severe hypercholesterolemia at high cardiovascular risk: a randomized, double-blind, placebo-controlled trial. J Am Coll Cardiol. 2013;62:2178-84.

68. Norata GD, Tibolla G, Catapano AL. PCSK9 inhibition for the treatment of hypercholesterolemia: promises and emerging challenges. Vascul Pharmacol. 2014;62:103-11. 